\title{
PARG dysfunction enhances DNA double strand break formation in S-phase after alkylation DNA damage and augments different cell death pathways
}

\author{
H Shirai ${ }^{1,4,5}$, AR Poetsch ${ }^{2,3,4,5}$, A Gunji ${ }^{2,5}$, D Maeda ${ }^{2,3,5}$, H Fujimori ${ }^{1}$, H Fujihara ${ }^{2,5}$, T Yoshida ${ }^{2,5}$, H Ogino ${ }^{2,3,5}$ and M Masutani ${ }^{*, 1,2,3,5}$
}

Poly(ADP-ribose) glycohydrolase (PARG) is the primary enzyme responsible for the degradation of poly(ADP-ribose). PARG dysfunction sensitizes cells to alkylating agents and induces cell death; however, the details of this effect have not been fully elucidated. Here, we investigated the mechanism by which PARG deficiency leads to cell death in different cell types using methylmethanesulfonate (MMS), an alkylating agent, and $\mathrm{Parg}^{-/-}$mouse ES cells and human cancer cell lines. Parg ${ }^{-/-}$mouse ES cells showed increased levels of $\gamma-\mathrm{H} 2 \mathrm{AX}$, a marker of DNA double strand breaks (DSBs), accumulation of poly(ADP-ribose), p53 network activation, and S-phase arrest. Early apoptosis was enhanced in Parg $^{-1-}$ mouse ES cells. Parg ${ }^{-/}$ES cells predominantly underwent caspase-dependent apoptosis. PARG was then knocked down in a p53-defective cell line, MIAPaCa2 cells, a human pancreatic cancer cell line. MIAPaCa2 cells were sensitized to MMS by PARG knockdown. Enhanced necrotic cell death was induced in MIAPaCa2 cells after augmenting $\gamma$-H2AX levels and S-phase arrest. Taken together, these data suggest that DSB repair defect causing S-phase arrest, but p53 status was not important for sensitization to alkylation DNA damage by PARG dysfunction, whereas the cell death pathway is dependent on the cell type. This study demonstrates that functional inhibition of PARG may be useful for sensitizing at least particular cancer cells to alkylating agents.

Cell Death and Disease (2013) 4, e656; doi:10.1038/cddis.2013.133; published online 6 June 2013

Subject Category: Cancer

Poly(ADP-ribose) glycohydrolase (PARG) is the primary enzyme responsible for de-poly(ADP-ribosylation) of proteins by cleaving the glycoside bonds of poly(ADP-ribose) (PAR)., Recent studies show that several PARG isoforms are produced by alternative splicing. ${ }^{3,4}$ The full-length $110-128 \mathrm{kDa}$ protein and a shorter $107 \mathrm{kDa}$ variant reside in the nucleus, whereas a $60 \mathrm{kDa}$ isoform is found in the mitochondria. ${ }^{3}$ Recently, ADP-ribosyl hydrolase-3 was also reported to cleave the glycoside bonds of PAR. ${ }^{5}$ In contrast to PARG, ARH3 also cleaves other substrates, such as acetylADP-ribose, ${ }^{6}$ and is mainly localized to the mitochondria. ${ }^{7}$ As PAR degradation after DNA damage is extensively inhibited in PARG knockout cells, ${ }^{4,8-11}$ PARG, but not ARH3, mainly mediates PAR degradation.

Like Poly(ADP-ribose) polymerase 1 (PARP-1), PARG is also recruited to DNA damage sites after ionizing irradiation. ${ }^{12}$ PARG-deficient mouse embryonic fibroblasts (MEFs) and mice lacking the full-length isoform show increased sensitivity to alkylating agents and $\gamma$-irradiation. ${ }^{8,13} \mathrm{Parg}^{-1-}$ ES cells generated by the disruption of exon 1 also show augmented sensitivity to $\gamma$-irradiation, alkylating agents, and cisplatin treatment. ${ }^{4}$ In addition, hydrogen peroxide treatment in PARG knockdown A549 cells delayed the repair of DNA single strand breaks (SSBs). ${ }^{14}$ On the other hand, mild resistance of PARG knockdown cells to hydrogen peroxide was reported in MEFs. ${ }^{15}$ PARG knockdown induced defects in DNA repair and mitotic checkpoints after $\gamma$-irradiation. ${ }^{16}$ $P A R G$ deficiency enhanced cell death induced by UV irradiation and $N$-methyl-N'-nitro- $N$ '-nitrosoguanidine (MNNG), an $\mathrm{SN}_{1}$-type alkylating agent, through augmented activation of apoptosis-inducing factor (AIF). ${ }^{17,18}$ AIF-induced apoptosis caused by oxidative stress is dependent on PARP-1-synthesized poly(ADP-ribose). ${ }^{19,20}$

These studies suggest that the effect of PARG inhibition is cell-type- and DNA damage-dependent. It is therefore important to elucidate the role of PARG in the responses induced by different DNA lesions and how PARG inhibition affects the cell death pathways in different cell types. Here we characterized the effects of the functional inhibition of PARG on the cell death pathway induced by the $\mathrm{SN}_{2}$-type alkylating agent, methylmethanesulfonate (MMS). The major DNA lesions caused by $\mathrm{SN}_{2}$-type alkylating agents are alkylation

\footnotetext{
${ }^{1}$ Division of Genome Stability Research, National Cancer Center Research Institute, Tokyo, Japan; ${ }^{2}$ Division of Biochemistry, National Cancer Center Research Institute, Tokyo, Japan and ${ }^{3}$ ADP-ribosylation in Oncology Project, National Cancer Center Research Institute, Tokyo, Japan

*Corresponding author: M Masutani, Division of Genome Stability Research, National Cancer Center Research Institute, 5-1-1 Tsukiji, Chuo-ku, Tokyo 104-0045, Japan. E-mail: mmasutan@ncc.go.jp

${ }^{4}$ These authors contributed equally to this work.

${ }^{5}$ Previous affiliation

Keywords: PARG; alkylating agent; cell death pathway; DNA strand break; S-phase arrest

Abbreviations: AIF, apoptosis-inducing factor; $\Delta \Psi$, mitochondrial membrane potential; DAPI, 4',6-diamidino-2-phenylindole; DDR, DNA damage response; DSB, double strand break; EdU, ethynyl deoxycytidine; MMS, methylmethanesulfonate; MNNG, $N$-methyl- $N$ '-nitro- $N$ '-nitrosoguanidine; ND1, NADH dehydrogenase-1; PAR, poly(ADP-ribose); PARG, poly(ADP-ribose) glycohydrolase; PARP-1, poly(ADP-ribose) polymerase 1; PI, propidium iodide; S $\mathrm{S}_{\mathrm{DS}}$, DNA-synthesizing; SSB, single strand break; ZVAD, Z-Val-Ala-Asp(Ome)-Fluoromethylketone

Received 12.7.12; revised 28.2.13; accepted 01.3.13; Edited by RA Knight
} 
of bases but not oxidative DNA lesions. The DNA damage induced by MMS includes 3-methyladenine lesions, which inhibit replication, as well as a comparatively smaller amount of $0^{6}$-methyl guanine lesions. DNA damage caused by MMS are mostly processed by mismatch repair, homologous recombination, and translesion repair processes. ${ }^{21}$ The results showed that PARG dysfunction increased DNA double strand breaks (DSBs) and enhanced S-phase arrest, both in p53 network active or inactive state, and augmented apoptosis and/or necrotic cell death depending on the cell type.

\section{Results}

Enhanced apoptosis in Parg $^{-1-}$ ES cells after MMS treatment. To address the effect of PARG deficiency on sensitization and cell death, we first used mouse ES cells, which were expected to exhibit normal cellular responses. In this study, we used $\mathrm{Parg}^{-/-}$ES cells, which show about $10 \%$ residual PARG activity. ${ }^{4}$ The growth of ES cells was not affected in the absence of DNA-damaging insults. ${ }^{4}$ In clonogenic survival assays, Parg $^{-/-}$ES cell clones experienced a 1.5-fold increase in cell death after MMS treatment (Figure 1a). Flow cytometry revealed a marked increase in the apoptotic sub-G1 cell population (cells with DNA content $<2 \mathrm{~N}$ ) in $\mathrm{Parg}^{-/-}$cells already by $12 \mathrm{~h}$ after treatment. In contrast, a slight increase was observed in $\mathrm{Parg}^{+/+}$cells only at $24 \mathrm{~h}$ (see Figure 1b). Oligonucleosomal DNA ladder formation was enhanced after MMS treatment in $\mathrm{Parg}^{-/-}$ES cells (Figure 1c). These results suggested that Parg deficiency enhances MMS-induced apoptosis in $\mathrm{Parg}^{-/-}$ES cells.

Enhanced S-phase arrest after MMS treatment induced by Parg deficiency. Flow cytometric analysis after PI (propidium iodide) staining (Figure 1b) suggested that S-phase arrest was enhanced in $\mathrm{Parg}^{-/-}$ES cells. This was confirmed by two-dimensional flow cytometry, which is able to discriminate between DNA-synthesizing $\left(\mathrm{S}_{\mathrm{DS}}\right)$ and DNA-synthesis-blocked S-phase $\left(\mathrm{S}_{\mathrm{BDS}}\right)$ populations by combining ethynyl deoxycytidine (EdU) incorporation and $\mathrm{PI}$ staining. Parg ${ }^{-1-}$ ES cells showed an increase in the $S_{\text {BDS }}$ population compared with wild-type ES cells $10 \mathrm{~h}$ after MMS treatment (Figure 1d).

Impaired PAR metabolism and decreased NAD levels induced by Parg deficiency. Parg deficiency led to impaired PAR degradation. Immunostaining showed a marked accumulation of PAR $1 \mathrm{~h}$ after MMS treatment in $\mathrm{Parg}^{-/-}$ES cells, but not in $\mathrm{Parg}^{+/+}$ES cells (Figure 1e). An increase in the poly(ADP-ribosylated) proteins was also detected in the nuclei $1 \mathrm{~h}$ after MMS treatment in $\mathrm{Parg}^{-1-}$ ES cells but not in wild-type cells (data not shown).

Then total PAR levels were measured by HPLC (Figure 1f, upper panel). Parg ${ }^{-/-}$cells exhibited an $\sim$ three-fold higher basal level of PAR than Parg $^{+/+}$cells $(P<0.05)$. Parg ${ }^{-/-}$ES cell clones demonstrated an additional 5-6-fold increase $1 \mathrm{~h}$ after MMS treatment, which persisted for up to $5 \mathrm{~h}$. In contrast, almost no change in PAR level was detected in $\mathrm{Parg}^{+/+}$ and Parg $^{+/-}$ES cells. The augmented basal PAR level in
$\mathrm{Parg}^{-/-}$cells was also confirmed by flow cytometry method (Supplementary Figure 1).

NAD levels in untreated cells were also higher in $\mathrm{Parg}^{-/-}$ ES cells than in $\mathrm{Parg}^{+/+}$ES cells $(P<0.05)$ (Figure 1f, lower panel). NAD levels did not change after MMS treatment of Parg $^{+/+}$and Parg $^{+/-}$ES cells; however, NAD levels decreased to one-fourth of that of untreated cells $1 \mathrm{~h}$ after MMS treatment in Parg $^{-/-}$ES cells $(P<0.05)$. On the other hand, ATP levels were not decreased and a tendency of transient increase $1 \mathrm{~h}$ after treatment was observed in all genotypes, which returned to almost normal levels $5 \mathrm{~h}$ after MMS treatment (Figure 1g). These results suggest that PAR accumulation and decreased NAD, but not changes in ATP levels, contribute to enhanced MMS-induced cell death in Parg $^{-/-}$ES cells. The Timm23, a survival regulator and the neighboring gene of $\mathrm{Parg}^{4}{ }^{4}$ was confirmed to be expressed under Parg deficiency (Supplementary Figure 2).

Enhanced DDR: Increased $\gamma \mathrm{H} 2 \mathrm{AX}$ foci and p53 network activation. $\gamma \mathrm{H} 2 \mathrm{AX}$ foci are used as a marker of DSBs. ${ }^{22}$ As shown in Figures $2 \mathrm{a}$ and $\mathrm{b}$, enhanced levels of $\gamma \mathrm{H} 2 \mathrm{AX}$ were observed in Parg ${ }^{-1-}$ ES cells compared with wild-type ES cells. This effect was even more pronounced at $1 \mathrm{mM}$ MMS. Western blot analysis of $\gamma \mathrm{H} 2 \mathrm{AX}$ revealed an enhanced timedependent increase in phosphorylation in $\mathrm{Parg}^{-/-}$ES cells, which persisted till $24 \mathrm{~h}$ after MMS treatment (Figure 2c).

The early DNA damage response (DDR) was also characterized by the activation of p53 signaling. Western blot analysis revealed that both p53 levels and the phosphorylation of Ser18, a p53-activating modification, increased earlier in $\mathrm{Parg}^{-/-}$ES cells (Figure 2c). Two targets of transcriptional co-activation by $\mathrm{p} 53$, namely Mdm2 and $p 21$, showed a tendency of increase in the mRNA levels in Parg $^{-1-}$ ES cells compared with wild-type cells (Figure 2d), and increased Mdm2 protein levels were also observed in $\mathrm{Parg}^{-/-}$ES cells $10 \mathrm{~h}$ after treatment (Figure $2 \mathrm{c}$ ), when both p53 protein levels and the phosphorylation level were highest.

Enhanced caspase-dependent cell death. Whereas caspase activation is a hallmark of apoptosis, calpains are proteases involved in PARP-1 and AIF-dependent cell death mechanisms. $^{23}$ Western blot analysis showed that, in contrast to wild-type ES cells, caspase-dependent cleavage of Parp-1 was evident in Parg ${ }^{-1-}$ ES cells $5 \mathrm{~h}$ after MMS treatment and peaked $10 \mathrm{~h}$ after treatment (Figure 2c).

Calpain cleavage of AIF could be associated with the release of AIF from the mitochondria in particular cases of PARP-1-dependent AIF cell death. The $57 \mathrm{kDa}$ cleaved form of $\mathrm{AIF}^{23}$ was not observed in any of the genotypes (data not shown).

A time-dependent enhancement in increased caspase activity was observed in Parg $^{-/-}$ES cells compared with wild-type ES cells (Figure 3a). Calpain activity increased 1.5-2.0-fold after MMS treatment, but no significant differences between wild-type and $\mathrm{Parg}^{-/-}$ES cells were detected (Supplementary Figure 3).

The involvement of caspases was further confirmed by monitoring MMS-induced cell death in the presence of the pan-caspase inhibitor, Z-Val-Ala-Asp(Ome)-Fluoromethylketone (ZVAD). As revealed by FACS analysis, ZVAD 


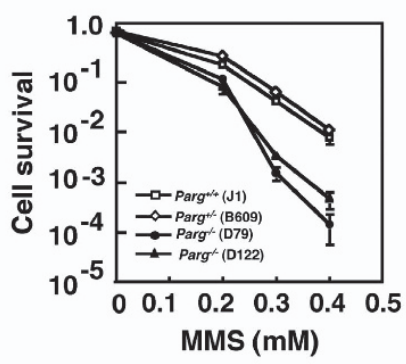

b
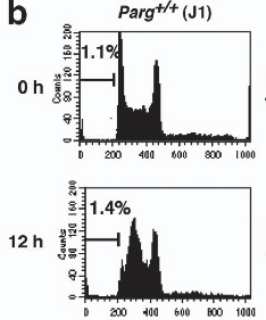

$24 \mathrm{~h}$

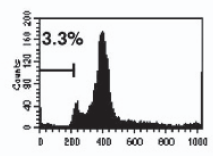

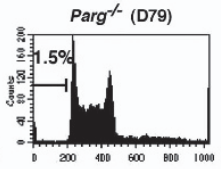
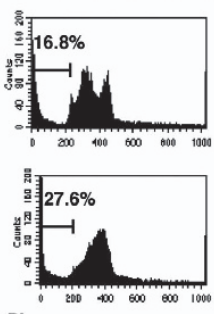
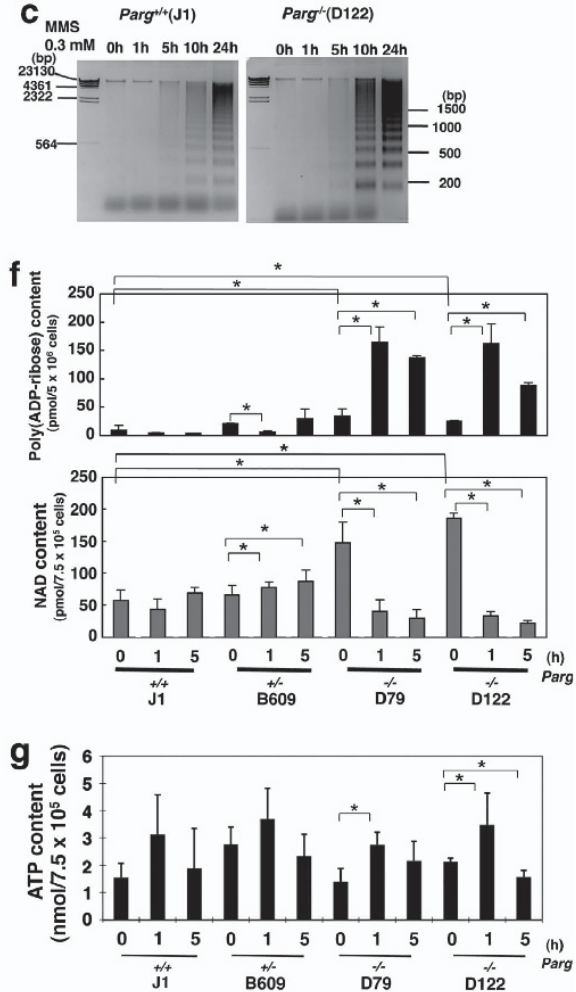

Figure 1 Enhanced cell death in Parg ${ }^{-1-}$ ES cells after MMS treatment. ES cells plated on STO feeder cells were treated with different concentrations of MMS. (a) Clonogenic survival assay. (b) Flow cytometric analysis of the apoptotic sub-G1 population and cell cycle distribution. In the absence of MMS treatment, cell cycle distribution was not altered by Parg deficiency. (c) Time course analysis of DNA ladder formation following the treatment with $0.3 \mathrm{mM}$ MMS. With this method, the fragmented DNA was solely recovered as described in the Materials and Methods. The fragmented DNA obtained from the same number of inoculated cells was subjected to electrophoresis. (d) Two-dimensional flow cytometry analysis of EdU incorporation and PI staining after MMS treatment. $\mathrm{S}_{\mathrm{DS}}$ corresponds to S-phase fraction showing DNA synthesis. $S_{B D S}$ corresponds to $S$-phase fraction showing blocked DNA synthesis. Percentage of the cells in $S_{B D S}$ fraction are marked with green line and their percentages are presented. (e) Immunostaining of ES cells with $10 \mathrm{H}$ antibody $1 \mathrm{~h}$ after treatment with $0.3 \mathrm{mM}$ MMS. Bar $40 \mu \mathrm{m}$. (f-g) Measurement of PAR and NAD (f), and ATP (g) levels in ES whole-cell extracts after treatment with $0.3 \mathrm{mM}$ MMS. PAR was extracted and fully digested by recombinant GST-Parg, ${ }^{36}$ and the amount of ADP-ribose was measured. Asterisks indicate statistically significant differences $(P<0.05)$. Untreated Parg ${ }^{+/+}$ES cells contained $\sim 9$ pmol of PAR (measured as the amount of ADP-ribosylated residues) per $5 \times 10^{6}$ cells, which is comparable to that reported in various cell types ${ }^{41}$

(20 and $50 \mu \mathrm{M}$ ) reduced enhanced cell death in $\mathrm{Parg}^{-/-}$ES cells after MMS treatment to the level of that in wild-type cells (Figure 3b). Cell death was also reduced in wild-type ES cells. These data suggest that Parg deficiency enhances caspase-dependent cell death.

Supplemental analysis showed that $\mathrm{Parg}^{-1-}$ ES cells were not further sensitized by PARP inhibitor PJ-34, suggesting that PARP inhibitor and PARG deficiency may dominantly act targeting the same pathway in ES cells (Supplementary Figure 4).

Parg deficiency affects plasma membrane integrity and the mitochondrial membrane potential. An early indicator of apoptosis is positive staining by Annexin V. The Annexin V-positive/PI-negative fraction was three times greater for Parg $^{-/}$cells than for wild-type cells 5 and $10 \mathrm{~h}$ after treatment (Figures $4 \mathrm{a}$ and $4 \mathrm{~b}$ ). The Annexin $\mathrm{V}$ and PI doublepositive fraction was slightly increased in $\mathrm{Parg}^{-/-}$ES cells (Figure 4c). The overall PI-positive fraction was clearly increased at $24 \mathrm{~h}$ in $\mathrm{Parg}^{-1-}$ ES cells (Figure 4d), which is characteristic of the late stages of cell death or necrosis.

A decrease in the mitochondrial membrane potential $(\Delta \Psi)$ is indicative of mitochondrial involvement during the execution of apoptosis ${ }^{24}$ and can be visualized by staining with $\mathrm{DIOC}_{6}$. After treatment with MMS, $\mathrm{DIOC}_{6}$ staining was attenuated in ES cells, regardless of Parg status (Figures $4 \mathrm{e}$ and f). No difference in the decrease of $\Delta \Psi$ was evident between the Parg genotypes in the PI-negative population. However, the decrease in $\Delta \Psi$ in the PI-positive population was enhanced in $\mathrm{Parg}^{-/-}$ES cells (Figures 4e and f), suggesting that mitochondrial involvement in cell death is enhanced by Parg deficiency.

Mitochondrial and genomic DNA fragmentation associated with apoptosis. The nature of the DNA damage was also analyzed by two-dimensional agarose gel electrophoresis. CAD, a DNase responsible for apoptosis-associated DNA cleavage, produces a diagonal ladder that results from double-stranded cleavage. There was an increase in double-stranded DNA fragmentation but no enhancement of the single-stranded DNA fragmentation was observed in the genomic DNA of $\mathrm{Parg}^{-/-}$ES cells (data not shown), suggesting that the DNase responsible for genomic DNA degradation might indeed be CAD, and that single-stranded genomic DNA formation is not involved for both genotypes. 


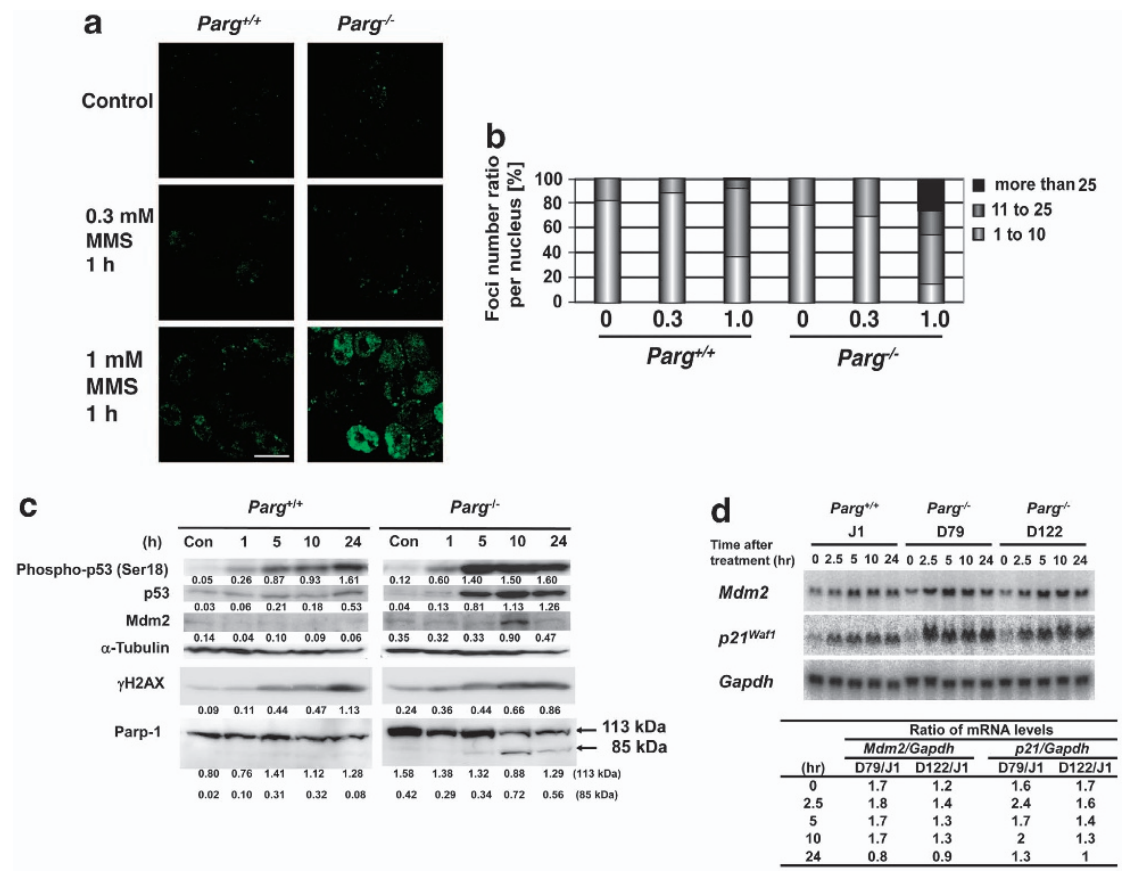

Figure 2 Early changes in the DDR after MMS treatment in Parg $^{-1-}$ ES cells. (a) Immunocytochemistry of $\gamma \mathrm{H} 2 \mathrm{AX}$ foci formation. Bar $15 \mu \mathrm{m}$. (b) The number of foci in ES cells was counted and quantified $1 \mathrm{~h}$ after treatment with $0.3 \mathrm{mM}$ MMS. (c) Western blot analysis of p53, mdm2, $\gamma \mathrm{H} 2 \mathrm{AX}$, and Parp-1. Western blot shows enhanced cleavage of Parp-1 in Parg ${ }^{-1}$ ES cells. The values under the bands show the intensities of the bands normalized by the band of $\alpha$-tubulin. Whereas caspases cleave Parp-1 to yield an 89-kDa C-terminal fragment, calpain-mediated cleavage of Parp-1 will produce a $40 \mathrm{kDa}$ N-terminal fragment. (d) Northern blot analysis of Mdm2 and p21waf1 expression. The lower panel shows mRNA levels normalized to Gapdh expression

Fragmentation of mitochondrial DNA was then examined using a probe for the mitochondrial NADH dehydrogenase-1 (ND1) gene (Figure 4g). Both double- and single-stranded DNA fragmentation increased in mitochondrial DNA after MMS treatment; however, an increase in double-stranded, but not single-stranded, DNA fragmentation was observed in $\mathrm{Parg}^{-1-}$ ES cells $5 \mathrm{~h}$ after MMS addition. This enhanced degradation of double-stranded DNA in mitochondria appears to parallel mitochondrial dysregulation and the enhanced reduction of $\Delta \Psi$ under Parg deficiency.

PARG knockdown enhances sensitivity to MMS in MIAPaCa2 and RKO cells. As the DDR may differ between cell types, siRNA knockdown was used to investigate the impact of $P A R G$ dysfunction in a human pancreatic cancer cell line, MIAPaCa2 (Figure 5a). MIAPaCa2 was chosen because the p53-dependent DDR pathway is inactivated, as frequently observed in human cancer. Clonogenic survival assays (Figure $5 \mathrm{~b}$ ) showed a dose-dependent increase in MMS-induced lethality by PARG knockdown in MIAPaCa2 cells $(P<0.05)$. We also compared the effect by $P A R G$ knockdown with PARP inhibitor PJ-34 and analyzed the combination effects of PARG knockdown and PJ-34. The sensitization effect by PARG knockdown was increased at the higher dose of MMS compared with PJ-34 alone, and their combination resulted in the enhanced sensitization.

We observed that PARG knockdown increased S-phase arrest $24 \mathrm{~h}$ after MMS treatment in MIAPaCa2 (Figure $5 \mathrm{c}$ ). Notably, the apoptotic sub-G1 population was observed in MIAPaCa2, but the level was not further enhanced by PARG knockdown. This suggested that $P A R G$ knockdown enhanced
MIAPaCa2 cell death through a pathway other than p53-dependent apoptosis. The levels of $\gamma \mathrm{H} 2 \mathrm{AX}$ protein and foci (Figures $5 \mathrm{~d}$ and e) were enhanced by MMS treatment after PARG knockdown. Nucleosome ladder formation associated with apoptosis was not detected (data not shown). When we analyzed with pulse-field gel electrophoresis, we observed an increase of necrosis-associated bands at around $50 \mathrm{~kb}$ (Supplementary Figure 5). Although p53 phosphorylation was not increased, histone $\mathrm{H} 3$ phosphorylation, a marker of the G2/M cell population, decreased in PARG knockdown cells compared with mock-treated MIAPaCa2, which is consistent with the observation of enhanced S-phase arrest (left panel, Figure 5e).

These results suggested that necrotic cell death, rather than apoptosis, was enhanced in MIAPaCa2. Western blot analysis revealed increased levels of HMGB1, a known indicator of necrotic cell death, ${ }^{25}$ in the culture medium of PARG knockdown MIAPaCa2 (left bottom panel, Figure 5e). When NAD levels were measured by HPLC, no change was detected after the addition of MMS (data not shown), suggesting that the decrease in NAD observed in mouse ES cells had not occurred in MIAPaCa2. PARP-1 cleavage, indicative of apoptotic cell death, was not observed in control or PARG knockdown cells (left panel, Figure 5e). These results suggest that $P A R G$ knockdown in MIAPaCa2 disturbs the DSB repair process after MMS treatment, resulting in enhanced necrotic cell death through S-phase arrest even under the defective p53 function, as shown in the model of Figure $5 f$.

We also compared the effect of PARG knockdown with PARP inhibitor PJ-34 and their combinations after MMS 

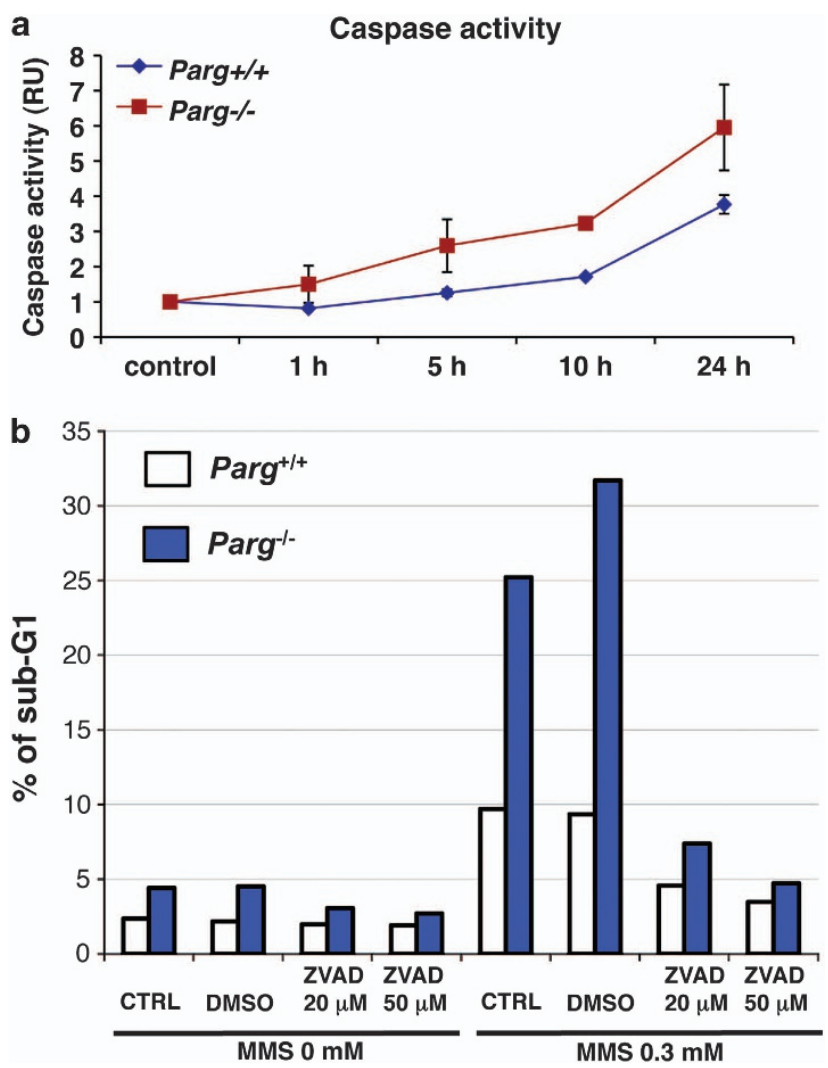

Figure 3 Enhanced caspase activity and suppression of enhanced cell death by caspase inhibition in $\mathrm{Parg}^{-1-}$ ES cells, following treatment with $0.3 \mathrm{mM}$ MMS. (a) Caspase activity. (b) Cell death in Parg ${ }^{-1}$ ES cells in the presence and absence of the caspase inhibitor ZVAD at 20 and $50 \mu \mathrm{M}$. Mean values of representative duplicate experiments are plotted

treatment on DDR signaling by western blot analysis (right panel, Figure 5e). It is notable that p53 and $\gamma \mathrm{H} 2 \mathrm{AX}$ phosphorylation were both attenuated by the combination of PARP inhibitor and PARG knockdown. DSB signaling possibly involving ATM kinase seems to be blocked by the combination of PARP inhibitor and PARG functional inhibition, and this may be dependent on cell types.

We further examined sensitization to MMS by $P A R G$ knockdown in another cancer cell line RKO, a colon cancer cell line that harbors functionally active p53. ${ }^{26}$ PARG knockdown also sensitized to MMS in RKO by clonogenic survival assay without enhancing subG-apoptotic fractions (Supplementary Figure 6).

\section{Discussion}

In this study, we observed that Parg dysfunction enhanced MMS-induced cell death in particular cell lines through different cell death pathways. In mouse ES cells, Parg deficiency enhanced caspase-dependent apoptosis through rapid accumulation of PAR, decreased NAD levels, increased $\gamma \mathrm{H} 2 \mathrm{AX}$ foci, p53 network activation, and S-phase arrest following MMS treatment. On the other hand, PARG knockdown in human MIAPaCa2 cells enhanced MMS-induced caspase-independent necrotic cell death through increased $\gamma \mathrm{H} 2 \mathrm{AX}$ foci and S-phase arrest, but not through enhanced p53 network activation and apoptosis. Similar sensitization effect by PARG knockdown to MMS was observed in human colon cancer cell line RKO. Therefore, our study suggested that p53-dependent response itself is not important for sensitization to alkylation DNA damage by PARG dysfunction. Increased DSBs and S-phase arrest under PARG dysfunction after MMS treatment are common processes that lead to cell death in both cell types.

Enhanced MMS-induced caspase-dependent apoptosis in Parg-deficient ES cells. In $\mathrm{Parg}^{-/-}$ES cells, we observed increased caspase activity and almost total suppression of enhanced cell death by the caspase inhibitor ZVAD. These observations indicate that Parg deficiency enhances caspase-dependent apoptosis in ES cells.

MMS is an $\mathrm{SN}_{2}$-type alkylating agent that causes DNA methylation of both nuclear and mitochondrial DNA. MMS is known to induce extensive SSBs through abortive mismatch repair in nuclear DNA, leading to PARP-1 hyperactivation in the nucleus. As expected, we observed the extensive accumulation of polyADP-ribosylated proteins in the nuclei of $\mathrm{Parg}^{-1-}$ ES cells, suggesting that PARG suppresses the accumulation of polyADP-ribosylated proteins mainly in the nucleus. In $\mathrm{Parg}^{-/-}$ ES cells, apoptotic DNA ladder formation was increased and observed earlier compared with the wild-type cells. This was accompanied by a parallel increase in mitochondrial DNA DSBs. These mitochondrial DSBs may be produced during the DNA repair process, and could be involved in the accelerated and augmented cell death caused by Parg deficiency.

In neuronal cells subjected to oxidative stress or the $\mathrm{SN}_{1}$-type alkylating agent MNNG, PAR induces translocation of mitochondrial AIF to nuclei to induce cell deaththrough the activation of calpains. ${ }^{19,27}$ Neither AIF cleavage to the active form $^{28}$ nor an increase in calcium-dependent calpain activity ${ }^{23}$ were observed in $\mathrm{Parg}^{-/-}$ES cells. As subcellular fractionation could not be carried out in ES cells, we could not exclude the possibility of enhanced translocation of AIF from mitochondria to nuclei, although we showed that caspase-dependent apoptosis was dominant in $\mathrm{Parg}^{-1-}$ ES cells after MMS treatment. No increase in the level of SSBs was observed in mitochondrial DNA in Parg $^{-1-}$ ES cells, indicating that AIF-dependent cell death may not be a major pathway of MMS-induced cell death in Parg $^{-1-}$ ES cells.

NAD level was increased at baseline in $\mathrm{Parg}^{-/-}$ES cells. This could be due to suppressed Parp-1 activity, which could result in preservation of NAD by the increased level of polyADP-ribosylated PARP-1 by Parg deficiency. As the decreased NAD and accumulation of PAR were observed both early and observed in parallel manner, these processes could be involved in the enhanced induction of apoptosis under Parg deficiency. In contrast, we observed that high ATP levels were maintained and that NAD levels were only approximately one-twentieth of that of ATP. Thus, the decrease in NAD levels in Parg ${ }^{-1-}$ ES cells did not cause a decrease in ATP. Therefore, enhanced cell death in Parg $^{-/-}$ ES cells does not follow the typical energy failure model of cell death. ${ }^{29}$ Although a decrease in NAD may perturb glycolysis and the TCA cycle in mitochondria, we speculate that the perturbation of glycolysis is not likely to be the primary cause of enhanced early apoptosis. 
a

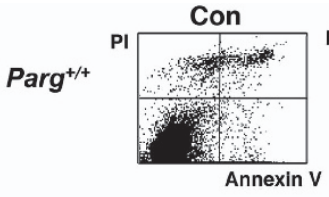

Parg-

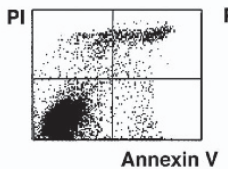

C
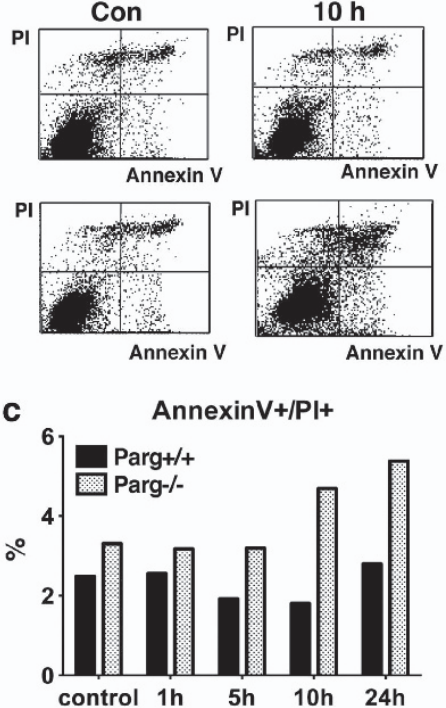

Annexin V b

d

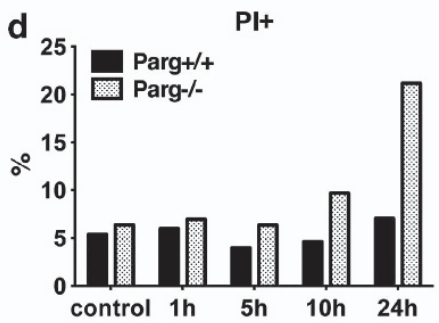

e
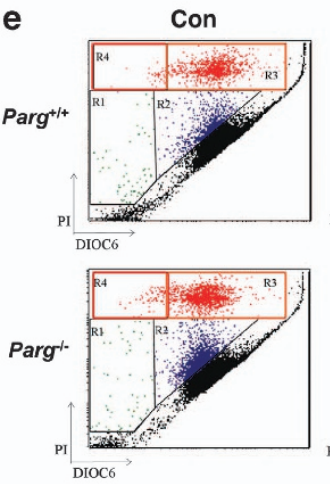
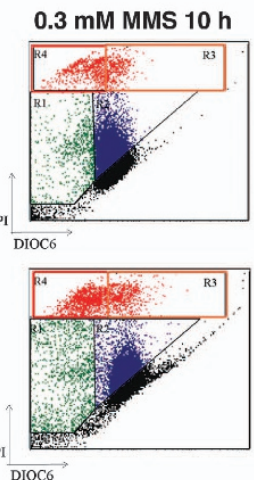

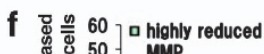
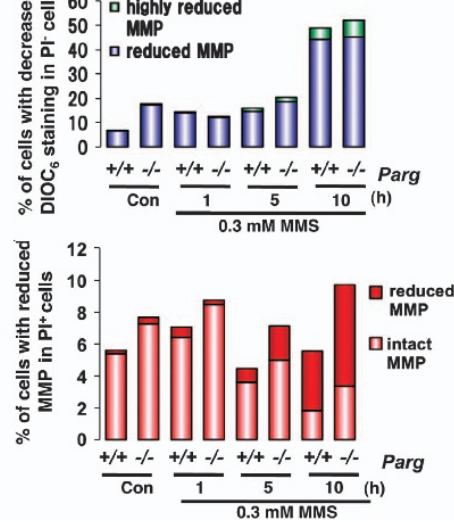

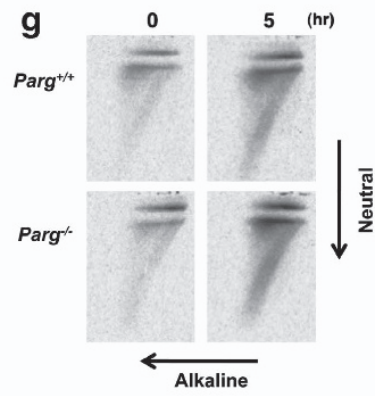

Figure 4 Early and late changes in plasma membrane and mitochondrial membrane potential in ES cells after treatment with $0.3 \mathrm{mM}$ MMS. (a) Time-course analysis of changes in plasma membrane integrity using propidium iodide (PI) and annexin V staining. Percentage of PI-positive (b), Annexin V-positive and PI-negative (c), and annexin V-positive and PI-positive fractions (d). Representative data are shown. (e and f) Mitochondrial membrane potential was measured by DIOC6 staining. (g) Two-dimensional electrophoresis is performed in one dimension under neutral conditions to analyze DSBs, and then in a second dimension under alkaline conditions to analyze single strand lesions. Fragmentation of mitochondrial DNA was then examined using a probe for the mitochondria-specific gene, ND1

A decrease in mitochondrial NAD levels triggers apoptosis under certain conditions. ${ }^{30}$ Although MMS causes NAD depletion from the nuclear and cytoplasmic pools, it is reported to show a lesser effect on the mitochondrial NAD pool due to the maintenance of NAD levels through the mitochondrial NAD salvage pathway. ${ }^{30}$ We observed that mitochondrial double-stranded DNA fragmentation was enhanced after MMS treatment in $\mathrm{Parg}^{-/}$ES cells, and it will be interesting to determine whether mitochondrial NAD levels are also decreased. As Parg deficiency caused extensive accumulation of polyADP-ribosylated proteins in the nuclei, nuclear PARP-1 may be extensively polyADPribosylated, which would inhibit its activity. ${ }^{8}$

PARG deficiency enhances caspase-independent MMS-induced necrosis in MIAPaCa2, not through enhanced p53 network activation. It is known that p53-dependent apoptosis is abrogated in MIAPaCa2. This cell line harbors a mutation in the p53 DNA-binding domain causing defective regulation of p53-dependent transcription. $^{31}$ MMS-induced phosphorylation of p53 was observed equally in PARG-knockdown MIAPaCa2 and controls. This suggests that p53 activation is not required for enhanced MMS-induced cell death by PARG knockdown. Release of the necrotic marker HMGB1 into the medium was clearly enhanced following PARG knockdown. Although SN1-type alkylating agents cause necrotic cell death through depletion of NAD and ATP, and activation of AIF, calpain, and cathepsin, ${ }^{23}$ we did not observe a decrease in NAD levels in either control or PARG knockdown after MMS treatment. Therefore, necrotic cell death may not be primarily triggered by a decrease in total cellular NAD levels.

Combination effect of PARP inhibitor and PARG functional inhibition. In MIAPaCa2, the sensitization effect by PARG knockdown was higher at the higher dose of MMS compared with PARP inhibitor alone. The combination of $P A R G$ knockdown and PARP inhibitor resulted in the enhanced sensitization in MIAPaCa2. It is notable that p53 and $\gamma \mathrm{H} 2 \mathrm{AX}$ phosphorylation was both attenuated by the 
a

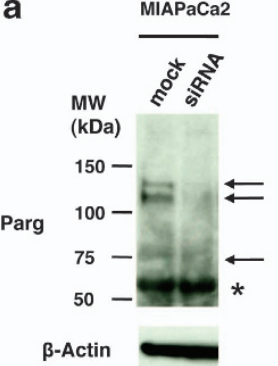

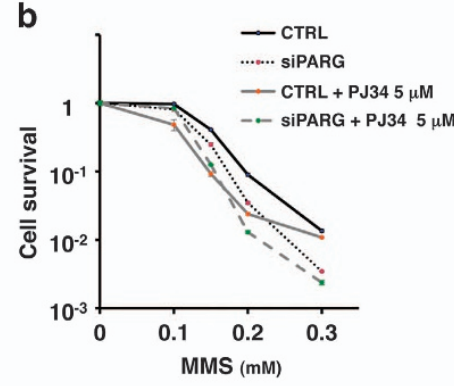
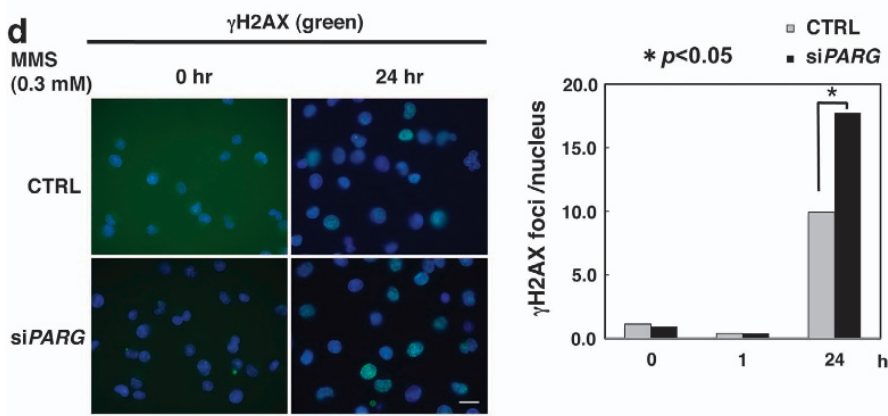

$\mathbf{C}_{\text {MMS }}$

(0.3 mM) SICTRL SIPARG
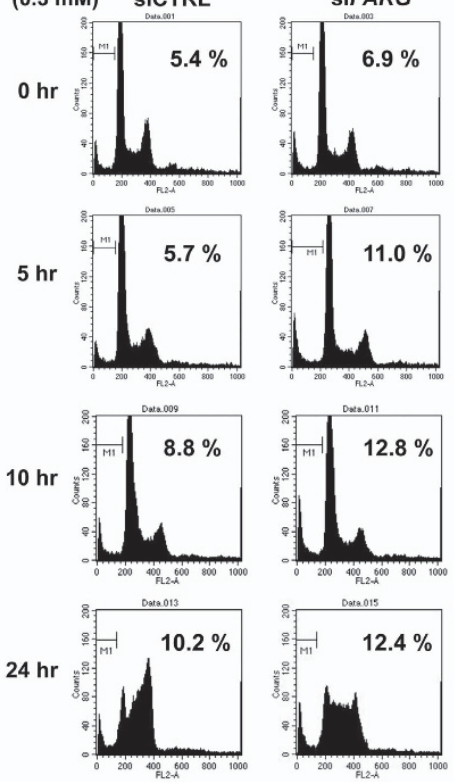

e

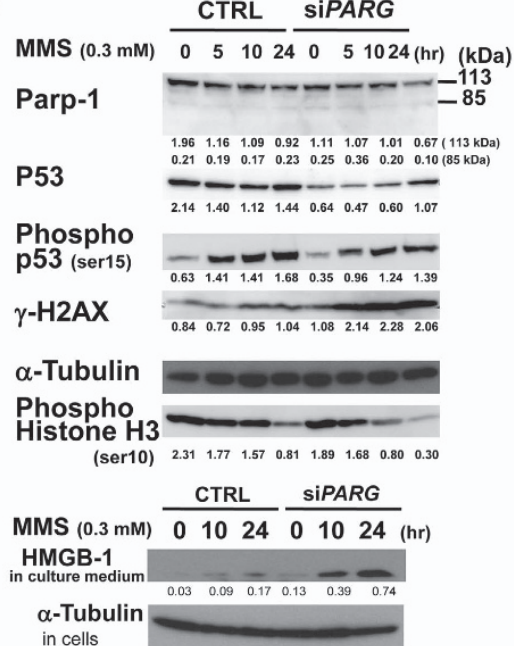

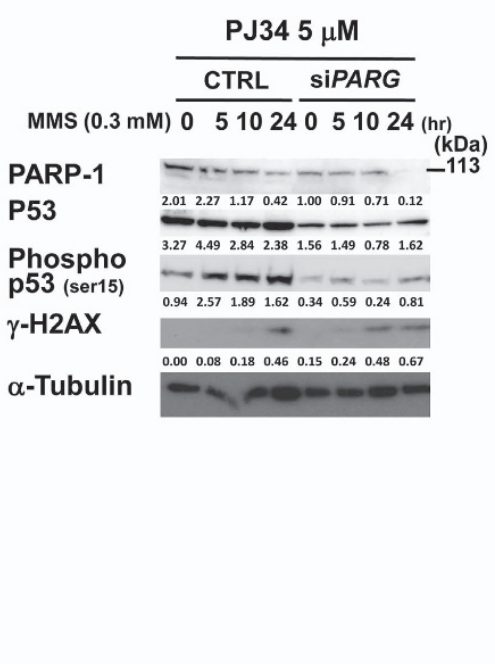

f

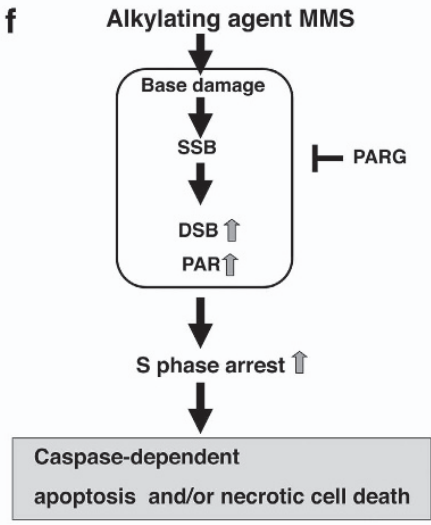

Figure 5 Enhanced sensitivity to MMS treatment in MIAPaCa2 induced by PARG knockdown. (a) Western blot analysis of PARG in MIAPaCa2. (b) Clonogenic survival assay of MIAPaCa2 after MMS treatment. We also compared the effect by PARG knockdown with PARP inhibitor PJ-34 at $5 \mu$ M and analyzed the combination effects of PARG knockdown and PJ-34. (c) Flow cytometric analysis of cell cycle distribution in MIAPaCa2. Percentages of sub-G1 fraction are shown. (d) Analysis of $\gamma \mathrm{H} 2 \mathrm{AX}$ foci formation in MIAPaCa2 after treatment with $0.3 \mathrm{mM} \mathrm{MMS}$. Bar $40 \mu \mathrm{m}$. (e) Western blot analysis of proteins involved in the DDR response (left panel) and necrosis (left panel, bottom part) after treatment with $0.3 \mathrm{mM}$ MMS in PARG knockdown MIAPaCa2. Combination effect of PARP inhibitor PJ-34 at $5 \mu \mathrm{M}$ was also analyzed for DDR response (right panel). The values under the bands show the intensities of the bands normalized by the band of $\alpha$-tubulin. (f) An induction model of different cell death pathways through common DSB increase and S-phase arrest in p53 active and defective cells. This study implies that p53-dependent response itself is not important for sensitization to alkylation DNA damage by PARG dysfunction

combination of PARP inhibitor and PARG knockdown, suggesting that PAR disturbs DSB repair. On the other hand, it also suggests that DSB signaling possibly by ATM kinase seems to be blocked by the combination of PARP inhibitor and PARG functional inhibition, leading to sensitization. Therefore, there is a clear-cell-type difference in the effect of combination of PARG deficiency and PARP inhibition.

Enhanced DSB repair defect and S-phase arrest were observed commonly in both Parg-deficient ES cells and MIAPaCa2 after MMS treatment. Levels of $\gamma \mathrm{H} 2 \mathrm{AX}$, a DSB marker, were enhanced in both Parg-deficient ES cells and $P A R G$ knockdown MIAPaCa2 after MMS treatment. MMS preferentially generates 3-methyladenine residues in DNA, which cause replication-fork arrest. ${ }^{32}$ An increase in S-phase arrest suggests that stalling of the replication fork was further enhanced by PARG deficiency. As SSB repair is reported to be delayed in Parg-deficient cells after hydrogen peroxide treatment, ${ }^{14}$ the SSB lesions may be preferentially converted to DSBs under PARG deficiency after MMS treatment. An increase in $O^{6}$-methylguanine lesions after MNNG treatment of Parg-deficient cells was also reported with enhanced 
decondensation of DNA. ${ }^{17,18}$ Therefore, the increase in DSBs after MMS treatment of cells with PARG dysfunction could be due to higher levels of alkylated bases and increased DNA accessibility. The increase in DSBs observed in cases of PARG dysfunction could also be associated with increased levels of stalled replication.

We noted that MIAPaCa2 and RKO both show defective mismatch repair, suggesting a possibility that $P A R G$ deficiency may induce sensitization to MMS through defective state of mismatch repair in these human cell lines. However, this could not be the case in ES cells, because mismatch repair system is active in the used wild-type and Parg-deficient ES cells.

Taken together, these results suggest that an S-phase block due to increased DSBs and PAR accumulation may be an important effect of PARG inhibition that enhances apoptosis and/or necrotic cell death in proliferating cell populations, as shown in Figure 5f. The results described in the present study imply that functional inhibition of PARG in combination with alkylating agents enhances either apoptosis or necrotic cell death through a defect in DSB repair and S-phase arrest.

\section{Materials and Methods}

Cell culture. All cell lines were cultured in a humidified atmosphere with $5 \%$ $\mathrm{CO}_{2}$ at $37^{\circ} \mathrm{C}$. ES cells were maintained in the absence of STO feeder cells and passaged every 3 days, as described elsewhere.

MIAPaCa2 cells were maintained in Dulbecco's Modified Eagle's Medium (Nissui, Tokyo, Japan) supplemented with 10\% FBS containing penicillinstreptomycin. The cells were passaged every 3 days in numbers appropriate to ensure logarithmic growth.

Clonogenic survival assay. Mouse Parg knockout ES cells ${ }^{4}$ and human $\mathrm{MIAPaCa} 2$ cells were cultured as described previously. ES cells were inoculated in triplicate onto a STO cell feeder layer in six-well plates (Iwaki Asahi Glass, Tokyo, Japan) and treated with MMS for $16 \mathrm{~h}$. They were then rinsed twice with $2 \mathrm{ml}$ of phosphate-buffered saline (PBS) and allowed to grow for 8 days. MIAPaCa2 cells were inoculated in triplicate in six-well plates (Nunc, Roskilde, Denmark) and treated with MMS for $16 \mathrm{~h}$. Cells were then washed twice with $2 \mathrm{ml}$ of PBS and cultured for 7 days. Colonies were stained with crystal violet for counting.

\begin{abstract}
Assessment of apoptosis. ES cells were harvested at different time points before and after treatment with $0.3 \mathrm{mM}$ MMS. For flow cytometric analysis, harvested ES cells were fixed, digested with RNase $\mathrm{A}$, stained with $\mathrm{PI}$, and analyzed using a BD FACSCalibur flow cytometer (BD Biosciences, San Jose, CA, USA). ${ }^{4}$ The effect of the pan-caspase inhibitor ZVAD (Enzo Life Science, Farmingdale, NY, USA) at 20 and $50 \mu \mathrm{M}$ was examined by flow cytometry. For DNA fragmentation analysis, the cells were suspended in lysis buffer (10 mM Tris-Cl (pH 7.4), $10 \mathrm{mM}$ EDTA, $0.5 \%$ Triton X-100), incubated on ice for $10 \mathrm{~min}$ and centrifuged at $17000 \mathrm{~g}$ for $20 \mathrm{~min}$. The supernatant was treated with RNase $A$ and proteinase $K$, and DNA was isolated by ethanol precipitation and analyzed by $2 \%$ agarose gel electrophoresis. With this method, the fragmented DNA was solely recovered. Two-dimensional gel electrophoresis was based on the study by Widlak et al. ${ }^{33}$ Electrophoresis in the first dimension was performed under Tris-Acetate-EDTA-buffered conditions at $4{ }^{\circ} \mathrm{C}$ for $4 \mathrm{~h}$ at $70 \mathrm{~V}$ using a pump. The gel was then soaked in alkaline buffer for $1 \mathrm{~h}$ and re-run under the same conditions apart from alkaline buffer and a time of $5 \mathrm{~h}$. The gel was neutralized with TAE for $1 \mathrm{~h}$, incubated with ethidium bromide solution, and analyzed using an LAS-3000 imaging system. The same gel was used for subsequent southern blotting with a probe for the ND1 gene ${ }^{34}$ isolated by PCR.
\end{abstract}

Two-dimensional flow cytometry with EdU and PI. To measure DNA synthesis, $3 \times 10^{5}$ cells were seeded into six-well dishes. MMS was added to the medium at a concentration of $0.3 \mathrm{mM}$, pulse-labeled with $50 \mu \mathrm{M}$ EdU (5-ethynyl-2-deoxyuridine, Molecular Probes, Molecular Probes, Eugene, OR, USA) for $1 \mathrm{~h}$, and harvested as described above. Detection of EdU incorporation was performed with the Click-iT EdU Alexa Fluor 488 Cell Proliferation Assay Kit (Molecular Probes), according to the manufacturer's instructions. In brief, $1 \times 10^{6}$ harvested cells were washed twice in PBS/ $1 \%$ BSA, fixed in $100 \mu$ l Click-iT fixative, and stored at $4{ }^{\circ} \mathrm{C}$. The following procedure was carried out for all the samples at the same time. After a $15 \mathrm{~min}$ incubation at room temperature in the dark, cells were washed twice in $1 \times$ saponin-based permeabilization and wash reagent. The Click-iT EdU reaction cocktail $(1 \times)$ was prepared according to the manufacturer's instructions and added to the cell pellet. Samples were incubated for $30 \mathrm{~min}$ at room temperature in the dark, and washed with $1 \times$ saponin-based permeabilization and wash reagent. For the staining of cellular DNA, samples were washed once in $1 \times$ saponin-based permeabilization and wash buffer. The DNA staining solution was prepared with $500 \mu \mathrm{l}$ saponin-based permeabilization and wash buffer, $0.2 \mathrm{mg} / \mathrm{ml} \mathrm{RNase}$ and $5 \mu \mathrm{g} / \mathrm{ml} \mathrm{PI}$. After $15 \mathrm{~min}$ at room temperature in the dark, cells were filtered through a $200 \mu \mathrm{m}$ mesh and analyzed using a BD FACSCalibur flow cytometer (BD Biosciences).

Flow cytometry instrumentation and data analysis. Univariate DNA staining, evaluation of apoptosis and EdU measurements were carried out on a standard BD FACSCalibur flow cytometer (BD Biosciences). Alexa Fluor 488 and PI signals were detected by FL1 and FL2 detectors, respectively. Sample measurements were performed using CellQuest Pro Software (BD Biosciences).

Measurement of PAR, NAD, and ATP contents in cells. Harvested cells were treated with $0.5 \mathrm{~N}$ perchloric acid and the acid-denatured samples were then neutralized with a mixture of potassium peroxide $(3 \mathrm{M})$ and glycine $(0.7 \mathrm{M})$ at $\mathrm{pH}$ 7.4. The supernatant was used for quantification of NAD and ATP by HPLC on a Develosil C30-UG-5 column $(46 \times 250 \mathrm{~mm}$, Nomura Chemicals, Seto, Japan). PAR was measured as the amount of ADP-ribose produced by the digestion of PAR. ${ }^{35}$ First, the insoluble fraction was treated with $1 \mathrm{~N}$ sodium hydroxide at $37^{\circ} \mathrm{C}$ for $1 \mathrm{~h}$, and then neutralized and digested with proteinase $\mathrm{K}$. After extraction with phenol and chloroform, and ethanol precipitation, the precipitate was digested with a glutathione $S$-transferase fusion protein of rat Parg (GST-Parg). ${ }^{36}$ The sample was treated with $0.5 \mathrm{~N}$ perchloric acid and neutralized, and the supernatant containing ADP-ribose produced from PAR was analyzed by HPLC as described above.

siRNA transfection. Transfection with siRNA was carried out using HiPerFect transfection reagent (Qiagen, Venlo, Netherlands) in six-well plates, as recommended by the manufacturer. Transfection solution consisting of $100 \mu \mathrm{l}$

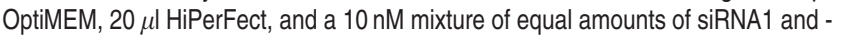
2 was used. (A combination of two different sequences is reported to reduce unintended knockdown effects). The following siRNA constructs were obtained from Ambion/Applied Biosystems (Austin, TX, USA):

siRNA1: 5'-AAGAAATGGGACTTTACAGCTTT-3' (exon 7), siRNA2: 5'-AAGAATAGAATCCATGATGAGTT-3' (exon 3).

A scrambled siRNA sequence was used as a negative control (Ambion/Applied Biosystems).

Northern blot analysis. Total RNA was prepared and $20 \mu \mathrm{g}$ of total RNA was electrophoresed under denaturing conditions in the presence of $2.2 \mathrm{M}$ formaldehyde, as described previously. ${ }^{36}{ }^{32}$ P-labeled mouse p21 and mdm2 probes used for northern blotting were prepared as described previously. ${ }^{37,38}$

Western blot analysis. Cells were extracted with Laemmli buffer, sonicated, and separated by SDS-polyacrylamide gel electrophoresis. The proteins were then transferred onto Sequi-Blot PVDF membranes (Bio-Rad, Hercules, CA, USA) and incubated with anti- $\alpha$-tubulin $(1: 5000$, ICN Biomedicals, Irvine, CA, USA), antihistone H3 (1:1000, Cell Signaling Technology, Danvers, MA, USA), antipoly(ADP-ribose) clone $10 \mathrm{H},{ }^{39}$ and anti-cytochrome oxidase subunit IV (cox 4) (1:5000, Molecular Probes) antibodies. Other antibodies used were anti-phospho (Ser15) p53 (1:1000, Cell Signaling Technology), anti-p53 (pan, 1:1000, Cell Signaling Technology), anti-mdm2 (1:1000, Cell Signaling Technology), anti-AIF (1:1000, Cell Signaling Technology), anti- $\gamma \mathrm{H} 2 \mathrm{AX}$ (1:5000, Upstate, Lake Piacid, NY, USA), anti-PARG (1:1000, Millipore, Temecula, CA, USA), and anti-HMGB1 (1:5000, Cell Signaling Technology). Immune complexes were visualized using a horseradish peroxidase-linked secondary antibody and enhanced chemiluminescence (Millipore).

FACS analysis of $\Delta \Psi$ and $\mathrm{PI}$ incorporation. This method is based on the principle that $\mathrm{DIOC}_{6}$ staining is dependent on mitochondrial $\Delta \Psi{ }^{24}$ Consequently, reduced staining with this dye indicates a decrease in $\Delta \Psi$. 
In parallel, $\mathrm{Pl}$ incorporation was observed to assess membrane function. Briefly, cells were trypsinized and washed with PBS, treated with $\mathrm{DIOC}_{6}(3)-\mathrm{PI}$ solution for $15 \mathrm{~min}$ at room temperature, and analyzed with a FACSCalibur flow cytometer (BD Biosciences). The areas of interest were gated and quantified.

Analysis of the effect of a pan-caspase inhibitor. A pan-caspase inhibitor ZVAD (Enzo Life Sciences) was dissolved in dimethyl sulfoxide, stored at $80^{\circ} \mathrm{C}$, and added to ES cell culture.

Measurement of caspase and calpain activity. In vitro caspase activity was assayed using the CaspACE Kit (Promega, Fitchburg, WI, USA) in a reaction mixture containing caspase buffer, $10 \mathrm{mM}$ dithiothreitol, and $200 \mathrm{mM}$ $\mathrm{N}$-Succinyl-Leu-Leu-Val-Tyr-AMC as a substrate following the manufacturer's protocol. At least $10^{6}$ cells per assay were used and the protein concentration was adjusted to $60 \mu \mathrm{g}$ protein per reaction.

The in vitro calpain assay was performed with the same lysates used for the caspase assay. Measurement was performed using the materials and protocol provided in the CaspACE Kit with some adjustments: $380 \mathrm{~nm}$ extinction and $460 \mathrm{~nm}$ emission wavelengths were used for analysis, and the emission was quantified.

Pulsed-field gel electrophoresis. Briefly, control and MMS-treated MIAPaCa2 cells were trypsinized and collected as described previously. ${ }^{40}$ The cells were immobilized in $0.75 \%$ agarose and treated with proteinase $\mathrm{K}(500 \mathrm{mM}$ EDTA, pH 9.0, $1.0 \% \mathrm{~N}$-lauroylsarcosine, $0.5 \mathrm{mg} / \mathrm{ml}$ proteinase $\mathrm{K}$ ) for $24 \mathrm{~h}$ at $50^{\circ} \mathrm{C}$ Electrophoresis was performed using a Bio-Rad CHEF-Mapper XA PFGE system. Samples were resolved in a $1 \%$ agarose gel at $5.4 \mathrm{~V} / \mathrm{cm}$ (Forward V-Gradient) and $3.6 \mathrm{~V} / \mathrm{cm}$ (Reverse V-Gradient) for $20 \mathrm{~h}$ with a 60 -s switch time ramp $\left(14^{\circ} \mathrm{C}\right)$. Gels were stained with ethidium bromide and photographed.

Immunofluorescence microscopy. For all fluorescence microscopy experiments, $4 \times 10^{5}$ cells were seeded $2 \mathrm{~h}$ prior to use in $35 \mathrm{~mm}$ glass base dishes (Iwaki, Asahi Glass). Cells were treated with $0.3 \mathrm{mM}$ MMS and incubated for 1 or $24 \mathrm{~h}$. The cells were washed with PBS and fixed in $4 \%$ formalin for $15 \mathrm{~min}$ at room temperature. Subsequently, the formalin reaction was quenched with $100 \mathrm{mM}$ glycine for $1 \mathrm{~min}$ at room temperature. Cells were washed twice with PBS and permeabilized with $0.2 \%$ Triton X-100 in PBS for 5 min. The cells were then washed once with PBS and processed according to the experiment. Nuclei were stained with DAPI (4',6-diamidino-2-phenylindole, 1:500 000 in PBS) for 15 min in the dark at room temperature. Cells were washed three times with PBS and analyzed using an Axiovert200 wide field microscope.

For immunological detection of $\gamma \mathrm{H} 2 \mathrm{AX}$ foci and PAR, the samples were blocked using Blockace for $1 \mathrm{~h}$ at room temperature. After washing twice with PBS, $250 \mu \mathrm{l}$ of the first antibody solution was added and the cells were incubated at $4{ }^{\circ} \mathrm{C}$ overnight. After washing with PBS three times, $250 \mu \mathrm{l}$ of the secondary antibody solution was added for $1 \mathrm{~h}$ in the dark at room temperature. Hoechst 33342 dye was added at $5 \mathrm{ng} / \mathrm{ml}$ for $10 \mathrm{~min}$. After washing three times with PBS, the sample was shielded with mounting medium and a coverslip. Images were taken using both an Axiovert200 wide field microscope and a confocal microscope.

Statistical analysis. Statistical analysis was performed using Mann-Whitney $U$-test and Student's $t$-test and SPSS (Macintosh version, SPSS, Chicago, IL, USA) or JMP (SAS Institute Inc., Cary, NC, USA) software.

\section{Conflict of Interest}

The authors declare no conflict of interest.

Acknowledgements. We are grateful for the suggestions provided by Alexander Bürkle, Shizuko Kobayashi, Fumiaki Koizumi, Sei-ichi Tanuma, David W Koh, Zhang Q Wang, Hitoshi Nakagama, and Takashi Sugimura. We thank for technical assistance by Hiromi Harada. This work was supported in part by a Grant-in-Aid for Cancer Research from the Ministry of Health, Labor and Welfare of Japan (19-9), a Grant-in-Aid from the Ministry of Health, Labor, and Welfare of Japan (11104962), the Third Term Comprehensive 10-Year Strategy for Cancer Control (10103833) from the Ministry of Health, Labor, and Welfare of Japan, from the MEXT of Japan $(15025274,22300343)$, and a Grant-in-Aid for Cancer Research from the Princess Takamatsu Cancer Research Fund.
1. Sugimura T. Poly(adenosine diphosphate ribose). Prog Nucleic Acid Res Mol Biol 1973: 13: 127-151.

2. Miwa M, Masutani M. PolyADP-ribosylation and cancer. Cancer Sci 2007; 98: 1528-1535.

3. Meyer-Ficca ML, Meyer RG, Coyle DL, Jacobson EL, Jacobson MK. Human poly (ADP-ribose) glycohydrolase is expressed in alternative splice variants yielding isoforms that localize to different cell compartments. Exp Cell Res 2004; 297: 521-532.

4. Fujihara $H$, Ogino $H$, Maeda D, Shirai $H$, Nozaki $T$, Kamada $N$ et al. Poly(ADP-ribose) Glycohydrolase deficiency sensitizes mouse ES cells to DNA damaging agents. Curr Cancer Drug Targets 2009; 9: 953-962.

5. Oka S, Kato J, Moss J. Identification and characterization of a mammalian $39-\mathrm{kDa}$ poly(ADP-ribose) glycohydrolase. J Biol Chem 2006; 281: 705-713.

6. Ono T, Kasamatsu A, Oka S, Moss J. The 39-kDa poly(ADP-ribose) glycohydrolase ARH3 hydrolyzes O-acetyl-ADP-ribose, a product of the Sir2 family of acetyl-histone deacetylases. Proc Natl Acad Sci USA 2006; 103: 16687-16691.

7. Niere M, Kernstock S, Koch-Nolte F, Ziegler M. Functional localization of two poly(ADP-ribose)-degrading enzymes to the mitochondrial matrix. Mol Cell Biol 2008; 28: 814-824.

8. Cortes U, Tong WM, Coyle DL, Meyer-Ficca ML, Meyer RG, Petrilli V et al. Depletion of the 110-kilodalton isoform of poly(ADP-ribose) glycohydrolase increases sensitivity to genotoxic and endotoxic stress in mice. Mol Cell Biol 2004; 24: 7163-7178.

9. Koh DW, Lawler AM, Poitras MF, Sasaki M, Wattler S, Nehls MC et al. Failure to degrade poly(ADP-ribose) causes increased sensitivity to cytotoxicity and early embryonic lethality. Proc Natl Acad Sci USA 2004; 101: 17699-17704.

10. Hanai S, Kanai M, Ohashi S, Okamoto K, Yamada M, Takahashi H et al. Loss of poly (ADP-ribose) glycohydrolase causes progressive neurodegeneration in Drosophila melanogaster. Proc Natl Acad Sci USA 2004; 101: 82-86.

11. Chambon $P$, Weil JD, Mandel P. Nicotinamide mononucleotide activation of a new DNA-dependent polyadenylic acid synthesizing nuclear enzyme. Biochem Biophys Res Commun 1963; 11: 39.

12. Mortusewicz $\mathrm{O}$, Fouquerel $\mathrm{E}$, Ame JC, Leonhardt $\mathrm{H}$, Schreiber V. PARG is recruited to DNA damage sites through poly(ADP-ribose)- and PCNA-dependent mechanisms. Nucleic Acids Res 2011; 39: 5045-5056.

13. Gao H, Coyle DL, Meyer-Ficca ML, Meyer RG, Jacobson EL, Wang ZQ et al. Altered poly(ADP-ribose) metabolism impairs cellular responses to genotoxic stress in a hypomorphic mutant of poly(ADP-ribose) glycohydrolase. Exp Cell Res 2007; 313: 984-996.

14. Fisher AE, Hochegger H, Takeda S, Caldecott KW. Poly(ADP-ribose) polymerase 1 accelerates single-strand break repair in concert with poly(ADP-ribose) glycohydrolase. Mol Cell Biol 2007; 27: 5597-5605.

15. Blenn C, Althaus FR, Malanga M. Poly(ADP-ribose) glycohydrolase silencing protects against H2O2-induced cell death. Biochem J 2006; 396: 419-429.

16. Ame JC, Fouquerel E, Gauthier LR, Biard D, Boussin FD, Dantzer F et al. Radiation-induced mitotic catastrophe in PARG-deficient cells. J Cell Sci 2009; 122 (Pt 12): 1990-2002.

17. Zhou Y, Feng X, Koh DW. Synergistic cytotoxicity of N-methyl-N'-nitro-N-nitrosoguanidine and absence of poly(ADP-ribose) glycohydrolase involves chromatin decondensation. Int J Oncol 39: 121-127.

18. Zhou $Y$, Feng $X$, Koh DW. Enhanced DNA accessibility and increased DNA damage induced by the absence of poly(ADP-ribose) hydrolysis. Biochemistry 49: 7360-7366.

19. Andrabi SA, Kim NS, Yu SW, Wang H, Koh DW, Sasaki M et al. Poly(ADP-ribose) (PAR) polymer is a death signal. Proc Natl Acad Sci USA 2006; 103: 18308-18313.

20. Koh DW, Dawson TM, Dawson VL. Mediation of cell death by poly(ADP-ribose) polymerase-1. Pharmacol Res 2005; 52: 5-14.

21. Schmidt KH, Viebranz EB, Harris LB, Mirzaei-Souderjani H, Syed S, Medicus R. Defects in DNA lesion bypass lead to spontaneous chromosomal rearrangements and increased cell death. Eukaryot Cell 9: 315-324.

22. Rogakou EP, Pilch DR, Orr AH, Ivanova VS, Bonner WM. DNA double-stranded breaks induce histone H2AX phosphorylation on serine 139. J Biol Chem 1998; 273 : 5858-5868.

23. Moubarak RS, Yuste VJ, Artus C, Bouharrour A, Greer PA, Menissier-de Murcia J et al. Sequential activation of poly(ADP-ribose) polymerase 1, calpains, and Bax is essential in apoptosis-inducing factor-mediated programmed necrosis. Mol Cell Biol 2007; 27: $4844-4862$.

24. Marzo I, Brenner C, Zamzami N, Susin SA, Beutner G, Brdiczka D et al. The permeability transition pore complex: a target for apoptosis regulation by caspases and bcl-2-related proteins. J Exp Med 1998; 187: 1261-1271.

25. Brusa D, Migliore E, Garetto S, Simone M, Matera L. Immunogenicity of 56 degrees $C$ and UVC-treated prostate cancer is associated with release of HSP70 and HMGB1 from necrotic cells. Prostate 2009; 69: 1343-1352.

26. Jang ER, Ryu M, Park JE, Kim JH, Lee JS, Song K. A new isoquinolinium derivative, Cadein1, preferentially induces apoptosis in p53-defective cancer cells with functional mismatch repair via a p38-dependent pathway. J Biol Chem 285: 2986-2995.

27. Yu SW, Andrabi SA, Wang H, Kim NS, Poirier GG, Dawson TM et al. Apoptosis-inducing factor mediates poly(ADP-ribose) (PAR) polymer-induced cell death. Proc Natl Acad Sci USA 2006; 103: 18314-18319. 
28. Ye H, Cande $\mathrm{C}$, Stephanou NC, Jiang S, Gurbuxani S, Larochette $\mathrm{N}$ et al. DNA binding is required for the apoptogenic action of apoptosis inducing factor. Nat Struct Biol 2002; 9 : 680-684.

29. Ha HC, Snyder SH. Poly(ADP-ribose) polymerase is a mediator of necrotic cell death by ATP depletion. Proc Natl Acad Sci USA 1999; 96: 13978-13982.

30. Yang $H$, Yang T, Baur JA, Perez E, Matsui T, Carmona JJ et al. Nutrient-sensitive mitochondrial NAD + levels dictate cell survival. Cell 2007; 130: 1095-1107.

31. Furuwatari C, Yagi A, Yamagami O, Ishikawa M, Hidaka E, Ueno I et al. A comprehensive system to explore p53 mutations. Am J Clin Pathol 1998; 110: 368-373.

32. Tercero JA, Diffley JF. Regulation of DNA replication fork progression through damaged DNA by the Mec1/Rad53 checkpoint. Nature 2001; 412: 553-557.

33. Widlak P, Garrard WT. Unique features of the apoptotic endonuclease DFF40/CAD relative to micrococcal nuclease as a structural probe for chromatin. Biochem Cell Biol 2006; 84: 405-410.

34. Moslemi AR, Darin N, Tulinius M, Wiklund LM, Holme E, Oldfors A. Progressive encephalopathy and complex I deficiency associated with mutations in MTND1. Neuropediatrics 2008; 39: 24-28.

35. Shirato M, Tozawa S, Maeda D, Watanabe M, Nakagama H, Masutani M. Poly(etheno ADP-ribose) blocks poly(ADP-ribose) glycohydrolase activity. Biochem Biophys Res Commun 2007; 355: 451-456.

36. Shimokawa T, Masutani M, Nagasawa S, Nozaki T, Ikota N, Aoki Y et al. Isolation and cloning of rat poly(ADP-ribose) glycohydrolase: presence of a potential nuclear export signal conserved in mammalian orthologs. J Biochem (Tokyo) 1999; 126: 748-755.
37. Masutani M, Nozaki T, Nishiyama E, Shimokawa T, Tachi Y, Suzuki H et al. Function of poly(ADP-ribose) polymerase in response to DNA damage: gene-disruption study in mice. Mol Cell Biochem 1999; 193: 149-152.

38. Masutani M, Nozaki T, Nishiyama E, Ochiya T, Wakabayashi K, Suzuki H, Sugimura T. Establishment of poly(ADP-ribose) polymerase-deficient mouse embryonic stem cell lines. Proc Japan Acad 1998; 74(Ser. B): 233-236.

39. Kawamitsu H, Hoshino H, Okada H, Miwa M, Momoi H, Sugimura T. Monoclonal antibodies to poly(adenosine diphosphate ribose) recognize different structures. Biochemistry 1984 23: 3771-3777.

40. Ogino HSA, Gunji A, Suzuki H, Nakagama H, Sugimura T, Masutani M. Agent-dependent effects of Parp-1 deficiency on DNA damage responses and genomic stability in mouse ES cells. In: Grier EV (ed) New Developments in Stem Cell Research. Nova Science Publishers, Inc.: New York, 2007. pp 133-147.

41. Affar EB, Shah RG, Poirier GG. Poly(ADP-ribose) turnover in quail myoblast cells: relation between the polymer level and its catabolism by glycohydrolase. Mol Cell Biochem 1999; 193: $127-135$.

\footnotetext{
(c) (i) $(-)$ Cell Death and Disease is an open-access journal published by Nature Publishing Group. This work is licensed under a Creative Commons Attribution-NonCommercialNoDerivs 3.0 Unported License. To view a copy of this license, visit http://creativecommons.org/licenses/by-nc-nd/3.0/
}

Supplementary Information accompanies this paper on Cell Death and Disease website (http://www.nature.com/cddis) 